\title{
Scleral topography and its usefulness in scleral lens fitting
}

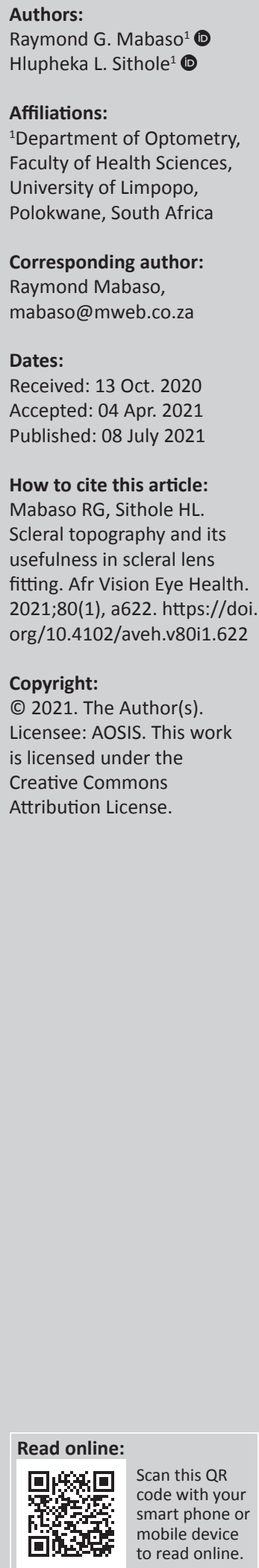

Background: Scleral lenses are one of the best options to manage corneal irregularities and severe ocular surface diseases. As scleral lenses rest on the sclera, scleral topographical data are necessary when fitting these lenses.

Aim: The aim of this article was to present a review of the literature on scleral topography and its usefulness in scleral lens fitting.

Method: A search was performed by the author with the PubMed database for the literature related to the topic by using a combination of keywords as search criteria. The most specific combination was represented by the junction of keywords (scleral topography and scleral lens fitting). Forty-four articles were related to scleral topography and scleral lens fitting.

Results: Fifteen articles were found to be more relevant to the topic and were selected for review. These articles originated from 11 different countries and five continents. Many (60\%) of the articles were from European countries.

Conclusion: The shape of the anterior surface of the eye beyond the limbus is not rotationally symmetrical, and the asymmetry increases as we move away from the limbus. Scleral topography is important in the fitting of scleral lenses, especially that keratometry measurements cannot be used to predict the base curve of the best fitting scleral lens.

Keywords: scleral lenses; topography; anterior scleral shape; scleral lens fitting; sagittal height; eye surface profiler; optical coherence tomography.

\section{Introduction}

The advent of scleral lens fitting appears to be on the rise in optometry settings in many countries, including South Africa. Interestingly, scleral lenses were the first contact lenses to be fitted on the human eye ${ }^{1,2}$ but were not widely prescribed. In recent years, scleral lenses have regained popularity as one of the best options for the management of corneal irregularities and some severe ocular surface diseases. ${ }^{1,3}$ The Scleral Lens Education Society defines a scleral lens as a rigid gas permeable (RGP) device that rests exclusively on the sclera and vaults the cornea and the limbus. These lenses are classified as mini-scleral when the lens diameter (LD) is up to $6 \mathrm{~mm}$ larger than the horizontal visible iris diameter (HVID) or large scleral lenses when the diameter is over $6 \mathrm{~mm}$ larger than the HVID. ${ }^{1}$ Because scleral contact lenses rest on the sclera and vault the cornea and the limbus, scleral topography becomes important in the fitting of these lenses.

Studies have shown that the nasal area of sclera is flatter than the temporal area, and that beyond the limbus, the sclera is not rotationally symmetrical with the asymmetry increasing with radial distance from the corneal apex., ${ }^{4,5}$ Because of their large diameter, scleral lenses provide good visual quality similar to that with RGP lenses as well as tolerance and comfort similar to that of soft contact lenses. ${ }^{6,7}$

The increase in the scleral lens market has led to an increase in the interest of eye care professionals in fitting these lenses. Many eye care professionals are familiar with the fitting process of corneal RGP lenses, which involves the use of corneal curvature as a starting point; however, scleral lens fitting does not require corneal curvature assessment because there is no contact between the lens and the cornea. Until recently, diagnostic sets were used in the fitting of scleral lenses. ${ }^{8}$ However, with advances in technology, topographical data that are important in scleral lens fitting, including the corneoscleral junction (CSJ), scleral curvature and angles, sagittal height and so forth, can now be accurately measured with optical coherence tomography (OCT) systems and other devices. The purpose of this article was to review the literature on scleral topography and its usefulness in scleral lens fitting. 


\section{Description of ocular surface parameters that are important in scleral lens fitting}

\section{Corneoscleral junction and corneoscleral angle}

The cornea meets the sclera at the CSJ. The shape of this junction may influence scleral lens design, fitting and potential patient comfort during scleral lens wear. The external angle that is formed between the line connecting the 10.0 and $12.8 \mathrm{~mm}$ chords and the line connecting the 12.8 and $15.0 \mathrm{~mm}$ chords, which may either be concave $\left(<180^{\circ}\right)$, tangential $\left(=180^{\circ}\right)$ or convex $\left(>180^{\circ}\right)$, is called the corneoscleral junction angle (CSA) (see Figure 1). ${ }^{9}$ The CSA varies with the ocular meridian examined, being sharpest nasally and flattest superiorly. However, the greatest asymmetry in the CSA is typically observed along the horizontal meridian, where the angle shows a sharp, concave transition nasally and a tangential or convex transition temporally. ${ }^{9,10}$ The difference between the nasal and temporal angles may lead to excessive limbal clearance, lens stand-off, lens decentration and conjunctival impingement in the temporal region. A toric or quadrant-specific lens design is recommended to achieve good lens alignment. ${ }^{11,12}$

\section{Limbal diameter}

The limbus is the border between the cornea and sclera. The approximate diameter of the limbus can be established by using the visible iris diameter as a reference point. Measuring the HVID and the vertical visible iris diameter (VVID) is important in contact lens fitting, particularly scleral lens fitting. Generally, the HVID is larger than the VVID, ${ }^{11,13}$ except in cases of against-the-rule corneal toricity. ${ }^{11,13}$ When the difference between the HVID and VVID is not clinically significant (not more than $0.5 \mathrm{~mm}$ ), the limbus is considered spherical and can be comfortably fitted with a spherical limbal zone scleral lens. However, when there is a significant difference (more than $0.5 \mathrm{~mm}$ ) between the HVID and VVID, the front view of the limbus will have a marked oval shape. Fitting a spherical lens on a marked oval shape limbus may result in lens decentration, conjunctival prolapse, discomfort and so forth. ${ }^{11}$ An overall elliptical or an oval trend scleral lens is required to achieve a comfortable and stable fit.

\section{Scleral curvature}

Studies have shown that the curvature of the anterior sclera is significantly steeper temporally compared with nasally, with substantially less variation between the nasal, superior and inferior quadrants. ${ }^{12,14,15}$ A steeper scleral curvature is associated with higher values of sagittal height (less elevated scleral surface), whereas the flatter curvature is associated with lower values of sagittal height (more elevated scleral surface). The scleral curvature varies with the ocular meridian examined, the distance from the limbus, age and so forth. ${ }^{12}$ Despite the marked variations of the scleral curvature between ocular meridians and the distance from the limbus, many patients are successfully fitted with rotationally symmetric lens designs. ${ }^{12}$

\section{The scleral angle}

The scleral internal angle is an angle that is formed at the junction of the $15.0 \mathrm{~mm}$ chord endpoint and a line along the scleral surface connecting the $12.8 \mathrm{~mm}$ and $15.0 \mathrm{~mm}$ chords (see Figure 1). ${ }^{9}$ The scleral angle is important with respect to scleral lens landing zone (LZ) alignment. Consequently, scleral lens manufacturers often specify adjustable tangent angles rather than peripheral curvatures to modify limbal clearance and the alignment of the LZ. The meridional differences (especially nasal-temporal asymmetry) in scleral angle tend to increase as we move away from the limbus,

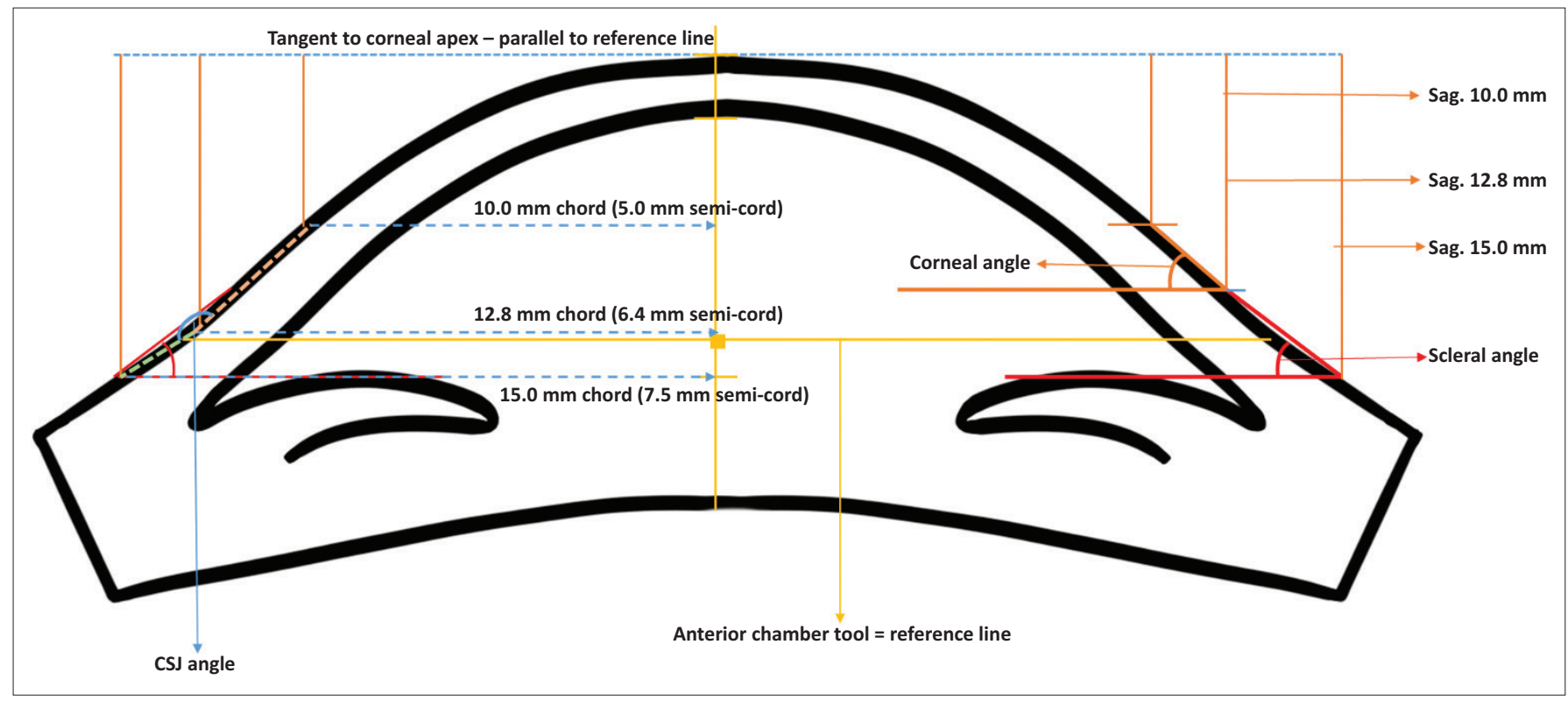

Source: Adapted from Ritzmann M, Caroline PJ, Börret R, Korszen E. An analysis of anterior scleral shape and its role in the design and fitting of scleral contact lenses. Contact Lens Anterior Eye. 2018;41(2):205-213. https://doi.org/10.1016/j.clae.2017.10.010

FIGURE 1: Sagittal height and corneoscleral angle measurements. 
particularly at chord length greater than $15 \mathrm{~mm}$. Therefore, spherical scleral lens with an LZ edge of about $15 \mathrm{~mm}$ or less should provide an adequate fit for most eyes; however, larger diameter lenses may require a toric or quadrant-specific haptic zone to achieve a comfortable, stable fit with an appropriate LZ alignment. ${ }^{9,12}$

\section{Sagittal height}

Sagittal height is intrinsically linked to the scleral angle. Sagittal height is the distance in micrometres $(\mu \mathrm{m})$ from the ocular surface at each chord length to a line tangent to the corneal apex and parallel to the reference line (see Figure 1). Therefore, ocular surfaces with lower sagittal height values are more elevated than those with higher sagittal height. Sagittal height varies with meridian examined, and the meridional differences in scleral elevation (scleral toricity) increase with increasing distance from the limbus (about $100 \mu \mathrm{m}$ at a $15-\mathrm{mm}$ chord to $400 \mu \mathrm{m}$ at a $20-\mathrm{mm}$ chord). ${ }^{9}$ As is the case with scleral angles, for smaller diameter scleral lenses (with an LZ of $15 \mathrm{~mm}$ or less), a rotationally symmetric lens design may be acceptable; however, for larger scleral lenses, customised haptic designs may be required to ensure lens stability with minimal decentration and lens flexure (particularly for thinner lenses and scleral toricity $\geq 200 \mu \mathrm{m}){ }^{12}$

\section{Methods}

A search was performed in the PubMed database for the literature related to the topic by using a combination of keywords as search criteria. The outcomes are presented in Table 1. The most specific combination was represented by the junction of keywords (scleral topography AND scleral lens fitting). Relevant articles were then analysed to determine whether they made any emphasis on scleral topography considerations and related usefulness in scleral lens fitting among various patients.

\section{Ethical considerations}

Ethical clearance was not required for this review. This article followed all ethical standards for research without direct contact with human or animal subjects.

\section{Results}

Forty-four articles were related to scleral topography and scleral lens fitting. Of these, 15 were found to be more relevant to the topic and were selected for review. Many (60\%) of the selected articles originated from European countries and one article (6.7\%) originated from South America (see Table 2).

\section{The features of the population studied in the reviewed studies}

The number of subjects in the reviewed studies ranged from 10 subjects $^{16}$ to 100 subjects. ${ }^{10}$ Most of the studies involved more women than men, except for one study that had more men than women. ${ }^{6}$ Most studies used subjects with healthy eyes. However, some studies included only subjects who
TABLE 1: Different combinations of searching criteria and the number of articles retrieved.

\begin{tabular}{lc}
\hline Search criteria & Number of articles retrieved \\
\hline Scleral topography & 212 \\
Scleral lens fitting & 204 \\
Scleral lens shape & 120 \\
Scleral topography and scleral lens fitting & 44 \\
\hline
\end{tabular}

were evaluated to receive their scleral lens prescriptions, ${ }^{5}$ those who experienced symptoms with their current lenses ${ }^{6}$ and those who were fitted with 18.2-mm Jupiter lenses to treat regular and irregular corneas. ${ }^{8}$ One study ${ }^{17}$ included more inclusion criteria than the others, from corneal ectasia, penetrating keratoplasty, post-surgical ectasia or regular corneas with myopia $\geq|6 \mathrm{D}|$, hyperopia $\geq|4 \mathrm{D}|$ and/or astigmatism.

Subjects with ocular diseases and history of ocular surgery were excluded in almost all the studies reviewed. In addition to this, subjects who were seen before by the researcher were excluded to eliminate the role of practitioner's experience on the study outcomes $;{ }^{18}$ subjects with connective tissue disease; ${ }^{19}$ pregnancy; $;^{17,19}$ and those who were contact lens users ${ }^{4,10,14,15}$ or had difficulty handling and caring for contact lenses, ${ }^{17}$ were excluded (see Table 2).

\section{Scleral topographers and other devices used to measure anterior ocular surface parameters}

Scleral topographers and OCT imaging devices are useful in the fitting of scleral lenses. The data from these devices are used to determine the first trial lens of choice and to objectively assess the central and peripheral lens fit and to customise lens design. Below is a brief description of some of the devices used in the reviewed articles.

\section{The Eye Surface Profiler (Eaglet Eye BV, the Netherlands)}

The Eye Surface Profiler (ESP) is a cornea and scleral topographer, which can measure an area of up to $20 \mathrm{~mm}$ in diameter with more than 250000 points, covering the rest of the cornea, the limbus and most part of the sclera (and conjunctiva). ${ }^{20}$ The ESP is based on the Fourier transform profilometry, which consists of two blue-light fringe projectors and a camera with a yellow filter positioned at the centre. To capture a good image of the anterior surface of the eye with the ESP, a viscous solution, such as Lubristil eye drops and sodium fluorescein, must be instilled into the eye. The ESP is a reliable and precise methodology to calculate the scleral radius and ESP may assist in estimating measurements for scleral lens fitting. ${ }^{20}$

\section{Medmont E300 corneal topographer (Medmont International Pty Ltd, Victoria, Australia)}

The Medmont E300 was used for corneal topography, estimated ocular surface sagittal height (OC-SAG) and scleral lens over-topography; ${ }^{9,16,18}$ corneal topography was measured by using the Medmont E300 corneal topographer; the Medmont Studio 6.1 was used to analyse the estimated OC-SAG for a chord value set by the examiner; ${ }^{9,18}$ and the 
TABLE 2: Sample size, gender and mean age of the subjects, and inclusion or exclusion criteria and country of publication of the reviewed studies

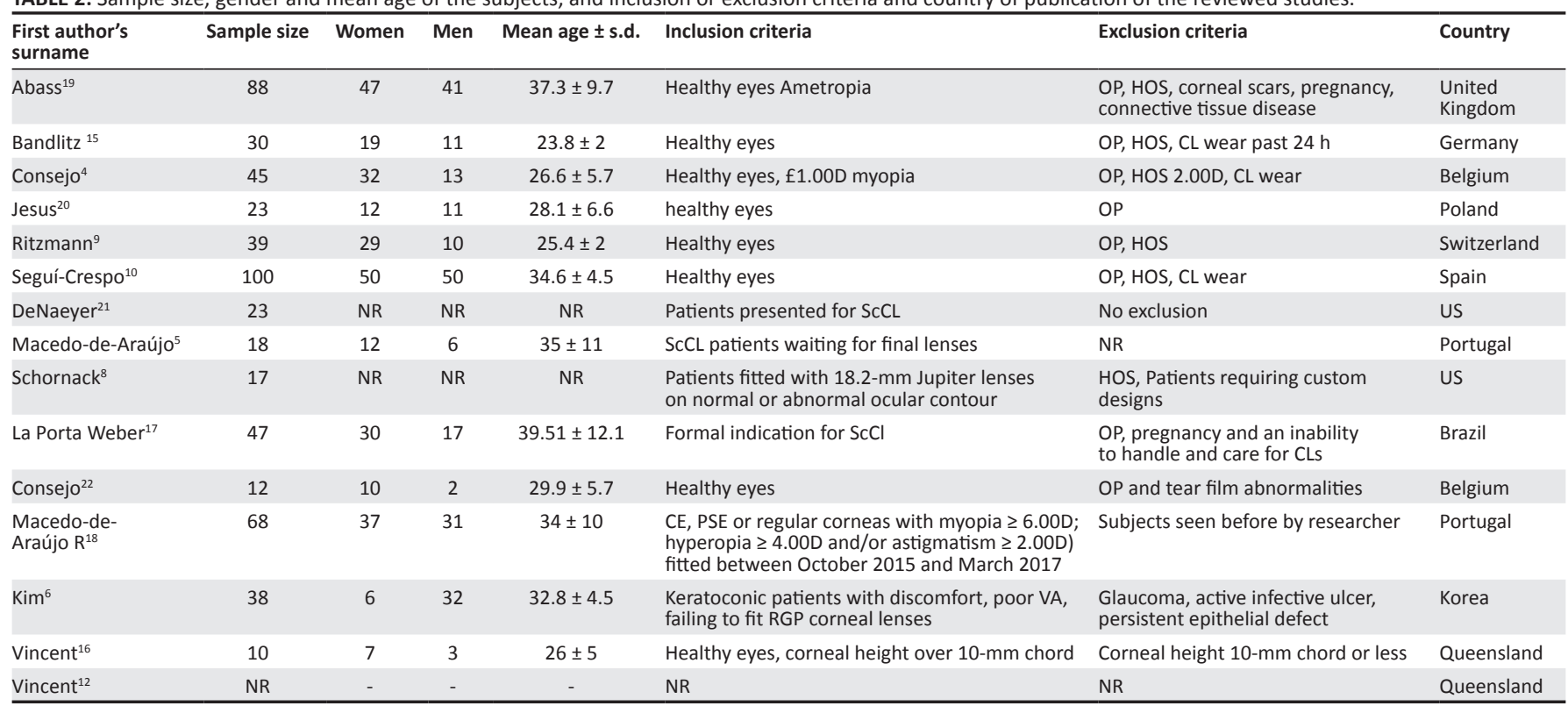

PK, penetrating keratoplasty; OP, ocular pathology; HOS, history of ocular surgery; NR, not reported; CE, corneal ectasia; PSE, post-surgical ectasia; ScCL, scleral contact lens; CL, contact lens; RGP, rigid gas permeable; s.d., standard deviation; US, United States.

Medmont E300 Placido ring videokeratoscope was used to measure scleral lens over-topography. ${ }^{16}$

\section{The Optos optical coherence tomography/scanning laser ophthalmoscope ${ }^{\mathrm{TM}}$ (Optos plc, Dunfermline, Scotland, United Kingdom)}

The Optos OCT $/ \mathrm{SLO}^{\mathrm{TM}}$ is a combination of spectral domain OCT imaging (SD-OCT) and a confocal scanning laser ophthalmoscope (SLO). An add-on lens is placed in front of the device optics to focus on the anterior segment of the eye. This device was used to measure the scleral radii in different orientations by asking subjects to look at the fixation targets along $0^{\circ}, 45^{\circ}, 90^{\circ}, 135^{\circ}, 180^{\circ}, 225^{\circ}, 270^{\circ}$ and $315^{\circ}$ in a randomised order. The SD-OCT provides an increased image acquisition speed and resolution, which allows for a clear description of anterior segment tissues. ${ }^{15}$

\section{The Fourier-domain optical coherence tomography system Copernicus HR (Optopol Technology \\ Sp. z.o.o., Zawiercie, Poland)}

The angular calliper of the software of the AS module of the Fourier domain OCT was used to measure CSA and the limbal scleral radii and the instrument provides faster acquisition times than time domain systems, and its higher resolution allows visualisation of greater detail. ${ }^{10,15}$

\section{The Visante optical coherence tomography (Carl Zeiss Meditec, Dublin, CA, USA)}

The Visante OCT is a time domain OCT, in which varying the position of the reference mirror of the device produces cross-sectional images. The device was used to measure the sagittal height of the anterior sclera and CSA in all eight primary meridians at chord lengths of 10.0, 12.8 and $15.0 \mathrm{~mm}$ and 12.8 and $15.0 \mathrm{~mm}$ to provide information regarding scleral topography. To achieve accurate measurement and comparison between the eight segments, the 'Anterior Segment Quad' scan was used to simultaneously image the four quadrants $\left(180^{\circ}, 45^{\circ}, 90^{\circ}\right.$ and $\left.135^{\circ}\right)$. The built-in calliper tool of the Visante OCT analysis software was used to measure the sagittal height and scleral angles by using landmarks on the anterior ocular surface as described above. ${ }^{9}$

\section{The IOLMaster $\mathbf{5 0 0}$ and $\mathbf{7 0 0}$ (Carl Zeiss Meditec AG, Jena, Germany)}

The IOL Master 500 has been the gold standard in optical biometry. The IOL Master 700 has been shown to be very similar to the IOL Master 500, although the IOL Master 700 was more effective in obtaining biometric measurements in eyes with posterior subcapsular and dense nuclear cataracts than the IOL Master 500.23 As mentioned above, this device was used to measure the axial length of the eye globe and other biometrical parameters such as corneal radius (CR), central corneal thickness (CCT), anterior chamber depth (ACD), white-to-white (WTW) corneal diameter and so forth. ${ }^{10,20}$

\section{The Pentacam rotating Scheimpflug (Oculus, Wetzlar, Germany)}

The Pentacam rotating Scheimpflug system uses a rotating Scheimpflug camera and a slit light source (blue LED at $475 \mathrm{~nm}$ ) that rotate together around the optic axis of the eye. Within $2 \mathrm{~s}$, the system rotates $180^{\circ}$ and captures from 12 to 50 Scheimpflug images (depending on the examiner's preference) that each contain 500 measurements for points on the front and back corneal surfaces and provide topography maps of these surfaces. The variables that can be assessed by the Pentacam include the anterior and posterior corneal elevation values, the corneal thickness at the thinnest point, the corneal volume, the $\mathrm{ACD}$, the anterior chamber angle and the corneal densitometry and lens sagittal depth (LSD) and LD..$^{10,17}$

It can be seen from the above that the ESP was the most commonly used device for corneoscleral topography of the eyes. $4,5,19,20,22$ The second most used devices included the 
Medmont E300 for corneal topography, which estimated ocular surface sagittal height (OC-SAG) and scleral lens over-topography, ${ }^{9,16,18}$ the OCT comprising two Fourier domain OCT to measure CSA and limbal scleral radii ${ }^{10,15}$ and one time domain OCT to measure the OC-SAG. The third most used devices were the Pentacam HR system to measure and analyse topography and tomography of the anterior segment of the eyes ${ }^{10,17}$ and the IOL Master 500 and 700 optical biometers to measure the axial length and other biometrical parameters of the eye. ${ }^{10,20}$ Seguí-Crespo et al. ${ }^{10}$ used more devices in their study than other studies in this review. The details of the other devices used and parameters measured are shown in Table 3.

\section{Discussion}

Most of the articles described the shape of the anterior ocular surface and how various measurements were made by using OCT and topographers. ${ }^{4,9,10,15,19,20,21,22}$ Some studies determined the relationship between corneal topographical data and scleral lens parameters. ${ }^{8,17,18}$ Other studies analysed the anterior ocular surface changes after scleral lens wear ${ }^{5,22}$ and another study quantified scleral lens decentration. ${ }^{12}$

Studies that described the shape of the anterior surface of the eye revealed that the overall sagittal height was higher on the

TABLE 3: Instrument name and parameters as measured.

\begin{tabular}{|c|c|c|}
\hline First author's name & Instrument name & Parameters measured \\
\hline Abass $^{19}$ & ESP & Corneoscleral topography \\
\hline \multirow[t]{2}{*}{ Bandlitz $^{15}$} & Optos OCT/SLO & LSR \\
\hline & Oculus Keratograph 4 & CCR, CE \\
\hline \multirow[t]{2}{*}{ Consejo $^{4}$} & ESP & Shape of sclera \\
\hline & Pentacam HR System & $\begin{array}{l}\text { Topographic and } \\
\text { Tomographic data } \\
\text { of the AS CSA }\end{array}$ \\
\hline \multirow[t]{3}{*}{ Jesus $^{20}$} & Fourier domain OCT & 3D topography of the AS \\
\hline & ESP & $\mathrm{AL}, \mathrm{CR}, \mathrm{CCT}, \mathrm{ACD}, \mathrm{WTW}, \mathrm{CD}$ \\
\hline & IOL Master 700 & $\begin{array}{l}\text { OC-SAG 10, 12.8, } 15 \mathrm{~mm} \\
\text { chord lengths }\end{array}$ \\
\hline \multirow[t]{2}{*}{ Ritzmann ${ }^{9}$} & Visante OCT & CSA \\
\hline & & Refraction \\
\hline \multirow[t]{3}{*}{ Seguí-Crespo ${ }^{10}$} & Fourier domain OCT & $\begin{array}{l}\text { Topographic and } \\
\text { tomographic data of the AS }\end{array}$ \\
\hline & VISIONIX VX120 & $\mathrm{AL}$ \\
\hline & Pentacam HR System & $\begin{array}{l}\text { OC-SAG; } 10,14,15,16,18, \\
20 \mathrm{~mm}\end{array}$ \\
\hline \multirow[t]{2}{*}{ DeNaeyer ${ }^{21}$} & $\begin{array}{l}\text { IOL Master } 500 \text { Optical } \\
\text { biometer }\end{array}$ & OC-SAG; $10 \& 12 \mathrm{~mm}$ \\
\hline & SMap3D & Topographic images \\
\hline \multirow{2}{*}{$\begin{array}{l}\text { Macedo-de-Araújo } \\
\text { Schornack }^{8}\end{array}$} & ESP & OC-SAG, CT, CD, ACD \\
\hline & Humphrey Atlas Topographer & Corneoscleral topography \\
\hline \multirow[t]{2}{*}{ La Porta Weber ${ }^{17}$} & $\begin{array}{l}\text { Pentacam Rotating } \\
\text { Scheimpflug Imaging System }\end{array}$ & OC-SAG; $14,15,16 \mathrm{~mm}$ \\
\hline & ESP & N/A \\
\hline Consejo ${ }^{22}$ & ESP & Scleral lens over-topography \\
\hline Macedo-de- Araújo ${ }^{18}$ & $\mathrm{~N} / \mathrm{A}$ & $\mathrm{N} / \mathrm{A}$ \\
\hline $\operatorname{Kim}^{6}$ & $\begin{array}{l}\text { Medmont E300, } \\
\text { videokeratoscope }\end{array}$ & - \\
\hline Vincent ${ }^{16}$ & N/A & - \\
\hline Vincent $^{12}$ & & \\
\hline
\end{tabular}

$\mathrm{AS}$, anterior segment; $\mathrm{ACD}$, anterior chamber depth; $\mathrm{AL}$, axial length; $\mathrm{CR}$, corneal radii; $\mathrm{CCT}$ central corneal thickness; CSA, corneoscleral angle; WTW, white-to-white diameter; CCR, central corneal radii; CE, corneal eccentricity; ESP, Eye Surface Profiler; LSR, limbal scleral radii; OC-SAG, ocular surface sagittal height; N/A, not applicable. temporal region than the nasal, for both right and left eyes. ${ }^{4,5,9,19}$ Macedo et al. ${ }^{5}$ reported that these findings were true for those eyes with regular or irregular corneas, although eyes with keratoconus had higher sagittal height than the eyes with regular corneas. ${ }^{5}$ Abass et al. ${ }^{19}$ indicated that the shape of sclera is not only steeper in the temporal region but also in two other meridians creating angles of nearly $120^{\circ}$ between them. In addition, the authors stated that the inferotemporal decentration of scleral lenses frequently noticed by practitioners may be because the nasal side of the sclera is higher than the temporal side. ${ }^{19}$ In addition to scleral shape, Ritzmann et al. ${ }^{9}$ indicated that gravity and eyelid forces during blinking may also be responsible for the scleral lens decentration. ${ }^{9}$

The shape of the eye in the limbal region (12.8-mm chord length) appears to be almost rotationally symmetric. ${ }^{9}$ However, beyond the limbus, the eye is rotationally asymmetric and the asymmetry increases progressively with an increase in chord length. 4,5,9,19 This scleral asymmetry was thought to be related to the effect of extraocular muscles' anatomy, where the shorter distance between the lateral and inferior rectus muscle insertions may create a flatter sclera nasally, whereas the greater distance between the medial and inferior rectus muscle insertions may contribute to a steeper scleral shape temporally., ${ }^{4,9}$ It is therefore important for optometrists interested in fitting scleral lenses to consider these parameters as they may have an influence on the comfort and wearing time as well as visual acuity of patients wearing them.

Studies that assessed the CSJs and scleral angles showed that the CSJ angle shows an abrupt, concave transition in the nasal region and a more convex and tangential transition in the temporal region. The scleral angles beyond the limbus were found to be flatter nasally than temporally, which would result in scleral lenses decentring temporally and inferiorly. ${ }^{9,10}$

The tangent angles were larger temporally than nasally for both regular and irregular corneas. ${ }^{5}$ Abass et al. ${ }^{19}$ reported that the CSJs were the sharpest at the nasal regions, and that there was a progressive flattening at the temporal, inferior and superior junctions. ${ }^{19}$ Bandlitz et al. ${ }^{15}$ revealed that the flattest scleral radius was in the superior nasal region and the steepest was in the temporal limbal region. ${ }^{15}$ This would have an influence on lens stabilisation on the eye. It has been shown that the higher the asymmetry between profiles, the more difficult it would be for lens to stabilise on the eye. ${ }^{10}$

A significant correlation between the axial length and scleral radius, ACD and scleral radius, and CR and the scleral radius was reported, indicating that a larger scleral radius would correspond with a larger CR for normal (emmetropic) eyes. ${ }^{20}$

Studies to determine the existence of a relationship between corneal topographic indices and scleral lens parameters 
showed that corneal topographic indices, especially in irregular corneas, cannot be used to choose the base curve of the scleral lenses that provide the most appropriate fit. A back-surface toric lens is required to have a proper fit in lenses with diameter larger than $15 \mathrm{~mm} .{ }^{8,18}$ The effect of short-term scleral lens wear on the anterior eye surface of the eyes was quantified in two of the reviewed studies, and both reported that there were no statistically significant changes on the limbal region and on the scleral conjunctiva, although there was steepening of the surfaces at $15-\mathrm{mm}$ and $16-\mathrm{mm}$ chord lengths, and slight alterations of the angles on the nasal regions. ${ }^{5,22}$ Scleral lenses were shown to decentre temporally and inferiorly in all the subjects. ${ }^{16}$ The review article summarised the potential use of OCT imaging in modern scleral lens practice including initial lens selection, assessing the fit and accurately quantifying the ocular response to lens wear. The understanding of anterior segment metrics including scleral thickness, curvature, toricity and the anatomy of the CSJ was discussed. ${ }^{12}$ Since many of these parameters were deduced mainly from patients in Western countries, it would be interesting to see the results of the parameters among an African-based population.

\section{Conclusion}

Direct comparisons of the study results and conclusions within this literature review are difficult, given the differences in the research designs, sample selection, subjects' characteristics, topographical devices used and so on. However, most of the studies that assessed the shape of the anterior surface of the eye agree that beyond the limbal region, the eye is not rotationally symmetrical, and that the asymmetry increases the further we move from the limbus.

There is also a general agreement that the nasal region of the sclera is flatter than the temporal region, resulting in the decentration of the lens temporally and inferiorly on the eye. It has been shown that keratometry measurements cannot be used to predict the base curve of the scleral lens that provides the most appropriate fit. These findings indicate the importance of scleral topography in scleral lens fitting. Because of the high cost of OCT imaging devices and scleral topographers, many optometrists from poor countries might not easily afford them, and therefore could struggle to fit scleral lenses as well as those who have the latest technologies.

\section{Acknowledgements Competing interests}

The authors declare that they have no financial or personal relationships that may have inappropriately influenced them in writing this article.

\section{Authors' contributions}

R.G.M. and M.L.S. contributed to the design and implementation of the research, to the analysis of the results and to the writing of the manuscript.

\section{Funding information}

No specific grant was received from any funding agency in the public, commercial or not-for-profit sectors.

\section{Data availability}

Data sharing is not applicable to this article.

\section{Disclaimer}

The views and opinions expressed in this article are those of the authors and do not necessarily reflect the official policy or position of any affiliated agency of the authors.

\section{References}

1. Van der Worp E, Bornman D, Ferreira DL, Faria-Ribeiro M, Garcia-Porta N, González-Meijome JM. Modern scleral contact lenses: A review. Contact Lens Anterior Eye. 2014;37(4):240-250. https://doi.org/10.1016/j.clae.2014.02.002

2. Pearson RM. Karl Otto Himmler, manufacturer of the first contact lens. Contact Lens Anterior Eye. 2007;30(1):11-16. https://doi.org/10.1016/j.clae.2006. 10.003

3. Tan DTH, Pullum KW, Buckley RJ. Medical applications of scleral contact lenses: 1. A retrospective analysis of 343 cases. Cornea [serial online]. 1995 [cited 2020 Apr 10];14(2). Available from: https://journals.Iww.com/corneajrnl/Fulltext/1995/ 03000/Medical_Applications_of_Scleral_Contact_Lenses_1_.1.aspx

4. Consejo A, Llorens-Quintana C, Bartuzel MM, Iskander DR, Rozema JJ. Rotation asymmetry of the human sclera. Acta Ophthalmol. 2019;97(2):e266-e270. https://doi.org/10.1111/aos.13901

5. Macedo-de-Araújo RJ, Van der Worp E, González-Méijome JM. In vivo assessment of the anterior scleral contour assisted by automatic profilometry and changes in conjunctival shape after miniscleral contact lens fitting. J Optom. 2019;12(2): 131-140. https://doi.org/10.1016/j.optom.2018.10.002

6. Kim S, Lee JS, Park YK, et al. Fitting miniscleral contact lenses in Korean patients with keratoconus. Clin Exp Optom. 2017;100(4):375-379. https://doi.org/10.1111/ cxo. 12424

7. Rosenthal P, Croteau A. Fluid-ventilated, gas-permeable scleral contact lens is an effective option for managing severe ocular surface disease and many corneal disorders that would otherwise require penetrating keratoplasty. Eye Contact Lens [serial online]. 2005 [cited $2020 \mathrm{Apr}$ 15];31(3). Availabel from: https:// journals.Iww.com/claojournal/Fulltext/2005/05000/Fluid_Ventilated,_Gas Permeable_Scleral_Contact.9.aspx

8. Schornack MM, Patel SV. Relationship between corneal topographic indices and scleral lens base curve. Eye Contact Lens [serial online], 2010 [cited $2020 \mathrm{Apr}$ 17];36(6). Available from: https://journals.Iww.com/claojournal/ Fulltext/2010/11000/Relationship_Between_Corneal_Topographic_Indices.3.aspx

9. Ritzmann M, Caroline PJ, Börret R, Korszen E. An analysis of anterior scleral shape and its role in the design and fitting of scleral contact lenses. Contact Lens Anterior Eye. 2018;41(2):205-213. https://doi.org/10.1016/j.clae.2017.10.010

10. Seguí-Crespo M, Ariza-Gracia MÁ, Sixpene NLD, Piñero DP. Geometrical characterization of the corneo-scleral transition in normal patients with Fourier domain optical coherence tomography. Int Ophthalmol. 2019;39(11):2603-2609. https://doi.org/10.1007/s10792-019-01109-5

11. Fadel D. The influence of limbal and scleral shape on scleral lens design. Contact Lens Anterior Eye. 2018;41(4):321-328. https://doi.org/10.1016/j.clae.2018.02.003

12. Vincent SJ, Alonso-Caneiro D, Collins MJ. Optical coherence tomography and scleral contact lenses: Clinical and research applications. Clin Exp Optom. 2019;102(3):224-241. https://doi.org/10.1111/cxo.12814

13. Iyamu E, Osuobeni E. Age, gender, corneal diameter, corneal curvature and central corneal thickness in Nigerians with normal intra ocular pressure. J Optom. 2012;5(2):87-97. https://doi.org/10.1016/j.optom.2012.02.001

14. Lee S-M, Choi HJ, Choi H, Kim MK, Wee WR. Estimation of axial curvature of anterior sclera: Correlation between axial length and anterior scleral curvature as affected by angle kappa. BMC Ophthalmol. 2016;16(1):176. https://doi.org/ 10.1186/s12886-016-0355-5

15. Bandlitz S, Bäumer J, Conrad U, Wolffsohn J. Scleral topography analysed by optical coherence tomography. Contact Lens Anterior Eye. 2017;40(4):242-247. https://doi.org/10.1016/j.clae.2017.04.006

16. Vincent SJ, Collins MJ. A topographical method to quantify scleral contact lens decentration. Contact Lens Anterior Eye. 2019:42(4):462-466. https://doi. org/10.1016/j.clae.2019.04.005

17. La Porta Weber S, Ambrósio Jr. R, Lipener C, Coral-Ghanem C, Hofling-Lima AL. The use of ocular anatomical measurements using a rotating Scheimpflug camera to assist in the Esclera" scleral contact lens fitting process. Contact Lens Anterior Eye. 2016;39(2):148-153. https://doi.org/10.1016/j.clae.2015.09.007 
18. Macedo-de-Araújo RJ, Amorim-de-Sousa A, Queirós A, Van der Worp E, GonzálezMéijome JM. Relationship of placido corneal topography data with scleral lens fitting parameters. Contact Lens Anterior Eye. 2019;42(1):20-27. https://doi. org/10.1016/j.clae.2018.07.005

19. Abass A, Lopes BT, Eliasy A, et al. Artefact-free topography based scleral-asymmetry PLoS One. 2019;14(7):e0219789. https://doi.org/10.1371/journal.pone.0219789

20. Jesus DA, Kedzia R, Iskander DR. Precise measurement of scleral radius using anterior eye profilometry. Contact Lens Anterior Eye. 2017;40(1):47-52. https:// doi.org/10.1016/j.clae.2016.11.003
21. DeNaeyer G, Sanders DR, Farajian TS. Surface coverage with single vs. multiple gaze surface topography to fit scleral lenses. Contact Lens Anterior Eye. 2017;40(3):162-169. https://doi.org/10.1016/j.clae.2017.03.009

22. Consejo A, Behaegel J, Van Hoey M, Wolffsohn JS, Rozema JJ, Iskander DR Anterior eye surface changes following miniscleral contact lens wear. Contact Lens Anterior Eye. 2019;42(1):70-74. https://doi.org/10.1016/j.clae.2018.06.005

23. Akman A, Asena L, Güngör SG. Evaluation and comparison of the new swept source OCT-based IOLMaster 700 with the IOLMaster 500. Br J Ophthalmol. 